\title{
Electromagnetic Time-Reversal Source Localization in Changing Media: Experiment and Analysis
}

Dehong Liu, Sathya Vasudevan, Jeffrey Krolik, ${ }^{1}$ Guillaume Bal and Lawrence Carin

Department of Electrical and Computer Engineering Duke University

Durham, NC 27708-0291

lcarin@ee.duke.edu

${ }^{1}$ Department of Applied Physics \& Applied Mathematics

Columbia University

New York, New York

\begin{abstract}
An experimental study is performed on electromagnetic time reversal in highly scattering environments, with a particular focus on performance when environmental conditions change. In particular, we consider the case for which there is a mismatch between the Green's function used on the forward measurement and that used for time-reversal inversion. We examine the degradation in the time-reversal image with increasing media mismatch, and consider techniques that mitigate such degradation. The experimental results are also compared with theoretical predictions for time reversal in changing media, with good agreement observed.
\end{abstract}

\section{Introduction}

Time reversal is a technique that is based on the principle of reciprocity [1]. In particular, assume a source emits radiation that propagates through a complex media to a set of receiving antennas. The data that arrives early in time at a given receiver implicitly travels a shorter distance than data that arrives later in time. By reversing the received waveforms in time, and emitting them from their respective reception points, the data that traveled a longer distance is emitted early, and the data that traveled a shorter distance is emitted later, and all of the energy arrives at the original source in unison, approximately recreating the original excitation. In the above discussion we have assumed that the medium is lossless. Further, the original source is not recreated exactly after time reversal, because in practice a finite set of receiver/source antennas are used. 
While the concept of time reversal is simple, a direct result of reciprocity, it has important implications. For example, in conventional imaging the focusing resolution is limited by the size of the antenna aperture (for an antenna array) [2]. However, in a complex propagation medium, characterized by multiple paths from the original source to the receiving elements, time reversal may have an effective aperture $[3,4]$ that is much larger than that of the physical array aperture, acting as a filter that increases with the number of available paths $[5,6]$. This phenomenon has been termed "superresolution" [4]. Interestingly, the more complex the media the more paths are manifested from a source to the multiple receivers, thereby enhancing superresolution refocusing quality (see Fig. 1). However, while resolution quality may be enhanced by increased media complexity, one also typically observes a reduction in the energy refocused at the original source $[4,5,6]$ (the complex media yields highly scattered waves, most of which are not observed by the antennas).

Time reversal has been demonstrated experimentally in an extensive set of ultrasonic and acoustic measurements [7-13], as well as in recent electromagnetic studies [14,15]. Time reversal examined in this previous work consists of two steps: (i) a source emits a pulse of radiation from a given point in a complex propagation environment, with the data observed at a set of receiving antennas; and (ii) the time-domain data at each of the receiver elements are reversed and synchronously re-radiated from the respective sourcereceiver antennas. A question arises as to focusing quality when there is a mismatch between the environments considered in steps (i) and (ii). This issue has been examined experimentally in acoustics $[8,16,17]$ and ultrasonics [10], as well as in theoretical studies [6]. Theory predicts that the strength of the refocused signal is a function of the correlation of the two underlying media between the forward and backward stages of the time reversal experiment.

We may seek to generalize time reversal, particularly when there is uncertainty in the medium associated with step (i). Addressing the imaging problem, rather than numerically implementing step (ii) through a single (fixed) media, which may be different from that actually used in step (i), we may perform this step using an ensemble 
of media (i.e., a media subspace, with the idea that the actual media from step (i) may be in the subspace, but without requiring knowledge of the exact/precise media associated with step (i)). For example, we may use an average Green's function for step (ii), with averaging performed over an ensemble of Green's functions corresponding to a set of (distinct) media related to the particular media for which step (i) was performed. Alternatively, one may perform an eigen-based principal components analysis (PCA) [18] on an ensemble of Green's functions for different media in step (ii), with use of the principal eigenvector(s) when imaging. We examine these techniques for electromagnetic source localization in changing and uncertain media.

\section{Theory of Time Reversal in Fixed and Changing Media}

\section{A. Superresolution}

A key property of time reversal sensing is that multiple scattering manifested by a complex (cluttered)

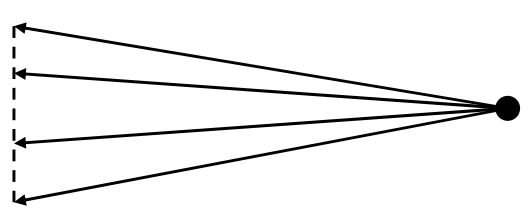

(a)

environment may be used to constitute an effective aperture that is larger than the actual physical aperture [4]. In Fig. 1 we consider a two-dimensional problem with a line-source radiator, with the fields observed along a linear aperture. In Fig. 1(a) the source radiates in a homogeneous medium, while in Fig. 1(b) the source radiates in a medium characterized by multi-path (clutter). Because of the homogeneous

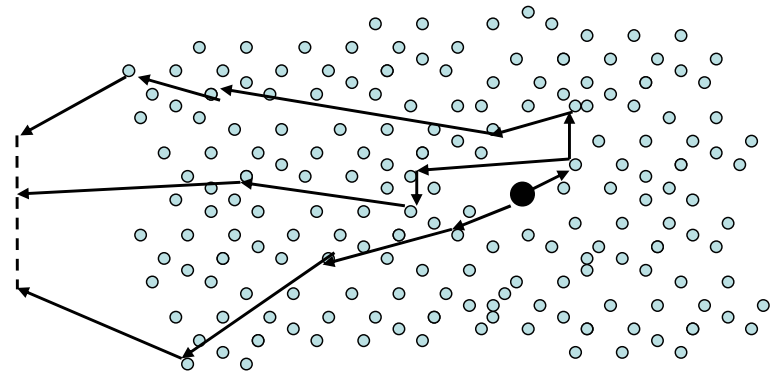

(b)

Figure 1. Wavefronts emitted from a source, observed at a linear antenna array (at left). (a) Source in a homogeneous medium, (b) in a highly multi-pathed environment. medium in which the fields radiate, in Fig. 1(a) the range of observed angle-dependent wavefronts is dictated by the size of the linear aperture and the distance of the source to the aperture [2]. By contrast, in Fig. 1(b) a richer set of waves emitted from the source 
make their way to the linear receiver aperture, as a result of multi-path. From Fig. 1 we therefore note that the number of different source wavefronts (emitted at different angles) observed by the receiver increases with increasing multi-path. When the data observed on the linear aperture is time reversed and synchronously reradiated, the multiple angledependent wavefronts emitted from the source are back-propagated, coalescing at the original source. Since the diversity of different angle-dependent waves arriving at the focus point increases with increasing clutter, the resolution (tightness) of the focus increases commensurately [1,4,7-14]; this has been referred to as "superresolution", because in a cluttered environment the focusing is tighter than would be expected in a homogeneous medium for the same physical aperture size.

\section{B. Rayleigh scattering}

In addition to examining the focusing tightness as a function of aperture size and clutter, we may examine the focusing energy strength as a function of frequency $f$. In the limit of small scatterer diameters with respect to the wavelength, it is known that scattering strength is proportional to $f^{4}$ for three-dimensional problems and to $f^{3}$ in two dimensions. This is Rayleigh scattering modeling light propagation in the atmosphere. We briefly recall how the scaling arises. Amplitude fluctuations in the wave speed caused by the presence of rods generates scatterers in the time harmonic representation (Lippman-Schwinger equation [19]) whose strength is proportional to the square of frequency [5]. Their power spectrum is therefore proportional to the fourth power of frequency. The homogeneous Green's function for observation point $\boldsymbol{r}$ and source point $\boldsymbol{r}^{\prime}$ may be expressed as

$$
G_{3 \mathrm{D}}\left(\boldsymbol{r}-\boldsymbol{r}^{\prime}\right)=\frac{e^{i\left|\boldsymbol{k} \| \boldsymbol{r}-\boldsymbol{r}^{\prime}\right|}}{4 \pi\left|\boldsymbol{r}-\boldsymbol{r}^{\prime}\right|}
$$

in three dimensions for wavenumber $|\boldsymbol{k}|=2 \pi f / c$, and therefore it has intensity independent of frequency, explaining the Rayleigh scaling in that setting (scattering proportional to $f^{4}$, and a Green's function with frequency-independent scaling). In two space dimensions, the Green's function is inversely proportional to the square root of frequency in the high frequency limit: 


$$
G_{2 \mathrm{D}}\left(\boldsymbol{r}-\boldsymbol{r}^{\prime}\right) \approx \frac{i}{4} \sqrt{\frac{2}{\pi k\left|\boldsymbol{r}-\boldsymbol{r}^{\prime}\right|}} e^{-i \pi / 4} e^{i k|\boldsymbol{x}|}, \quad k\left|\boldsymbol{r}-\boldsymbol{r}^{\prime}\right|>>1 .
$$

The resulting intensity is thus inversely proportional to frequency. The power spectrum is proportional to $f^{4}$ and the energy free propagator is proportional to $f^{-1}$, and the product of these two provides the $f^{3}$ Rayleigh scaling in two space dimensions.

\section{Correlations}

Time reversal as viewed in the frequency domain is the product of two Green's function propagators: the first one sends the signal from the source to the array of detectors, and the second one backpropagates the signal from the detector array. In the above discussion we assumed that the media associated with the forward and inverse phases were the same, and therefore that the respective Green's functions were identical; this represents the conditions under which time reversal is typically implemented [1]. It is of interest to examine the time-reversal image quality when the forward and inverse media are different (e.g., because the media conditions have changed, or because an approximate numerical Green's function is used in the inverse problem for representation of the actual Green's function associated with a forward measurement).

For the case of different Green's functions in the forward and inverse phases, the product of operators has a kernel represented by the correlation function of the two corresponding Green's function. In the Lippman-Schwinger representation of the wave equation [19], the correlation of the two Green's functions turns out to be proportional to the power spectrum of the random heterogeneities of the underlying heterogeneous media. If we denote by $\delta c(\mathbf{x})$ the fluctuations in the propagation speed of the underling medium, relative to a reference homogeneous medium, the correlation function as a function of position $\boldsymbol{x}$ is defined by

$$
R(\boldsymbol{x})=\langle\delta c(\boldsymbol{y}) \delta c(\boldsymbol{x}+\boldsymbol{y})\rangle,
$$

which is independent of $\boldsymbol{y}$ when the fluctuations are statistically independent by translation. The power spectrum $\hat{R}(\mathbf{k})$ is defined as the Fourier transform of the above correlation. 
When the underlying medium does not change between the two phases of the time reversal experiment, it is well known that the correlation of the two Green's function solves a radiative transfer equation in the phase space [19]. When the two media are different, then the radiative transfer equation needs to be modified. The salient feature of this modification is the following: the scattering coefficient in the radiative transfer equation, which was proportional to the power spectrum of the heterogeneities, is now proportional to the cross-correlation of the two sets of heterogeneous fluctuations; see [6]. When $\delta c_{j}(\boldsymbol{x})$ are the speed fluctuations during states $j=1$ (forward stage) and $j=2$ (backward stage) of the time reversal experiment, the cross-correlation is given by $R_{12}(\boldsymbol{x})=\left\langle\delta c_{1}(\boldsymbol{y}) \delta c_{2}(\boldsymbol{x}+\boldsymbol{y})\right\rangle$.

The analysis in [5] shows that the intensity of the time reversed signal is all the more important when the medium is scattering, i.e., heterogeneous. Consequently, when the two random media become less and less correlated, the scattering term in the radiative transfer equations decreases and so does the intensity of the time reversed signal. Such decay was validated by numerical studies in [5].

\section{Shifted media}

The above discussion considered the general case of changing media in the forward and inverse phases. We now consider the special case for which the media in the inverse phase is shifted by the vector $\tau$ with respect to the media considered in the forward measurement. As media separate from one another, the power spectrum appearing in the radiative transfer equations is multiplied by a plane wave (complex exponential) in the direction of the shift. Indeed we observe that

$$
R_{12}(\boldsymbol{x})=\left\langle\delta c_{1}(\boldsymbol{y}) \delta c_{2}(\boldsymbol{x}+\boldsymbol{y})\right\rangle=\left\langle\delta c_{1}(\boldsymbol{y}) \delta c_{1}(\boldsymbol{x}+\boldsymbol{\tau}+\boldsymbol{y})\right\rangle=R(\boldsymbol{x}+\boldsymbol{\tau}) .
$$

This shift by $\tau$ in the physical domain translates to multiplication by $e^{i \tau \cdot k}$ for the power spectrum in the Fourier domain: $\hat{R}_{12}(\mathbf{k})=e^{i \tau \cdot \mathbf{k}} \hat{R}(\mathbf{k})$. 
In the diffusive regime of wave propagation, where the energy of waves propagating in all directions is equipartitioned, this complex exponential is averaged over all directions of propagation [5]. The integral of plane waves over the space of directions

$\frac{1}{4 \pi} \int_{S^{2}} e^{i k \hat{\mathbf{k}} \cdot \tau} d \hat{\mathbf{k}}=\frac{\sin (k \tau)}{k \tau}, \frac{1}{2 \pi} \int_{S^{1}} e^{i k \hat{\mathbf{k}} \cdot \tau} d \hat{\mathbf{k}}=J_{0}(k \tau)$

provides a sinc function in three dimensions and a Bessel function in two dimensions, where $\tau=|\boldsymbol{\tau}|$. In either case, it can be identified with the imaginary part of the Green's function in homogeneous media.

The strength of scattering is multiplied by the Bessel function $J_{0}(k \tau)$ in the case of twodimensional shifted media considered below. In the diffusive regime, we thus obtain that the refocused signal vanishes for those frequencies such that $k \tau$ is a zero of the Bessel function; see [5] for more details.

\section{E. Accounting for mismatched media}

The discussion in Secs. IIC and IID demonstrates that the time-reversal imaging quality is expected to deteriorate with diminished correlation between the Green's functions used in the forward and inverse measurements. However, this was based on the assumption that we only had access to a single (mismatched) Green's function for the inverse phase. In Sec. V we extend these ideas by considering the case for which we assume access to an ensemble of Green's functions, and it is

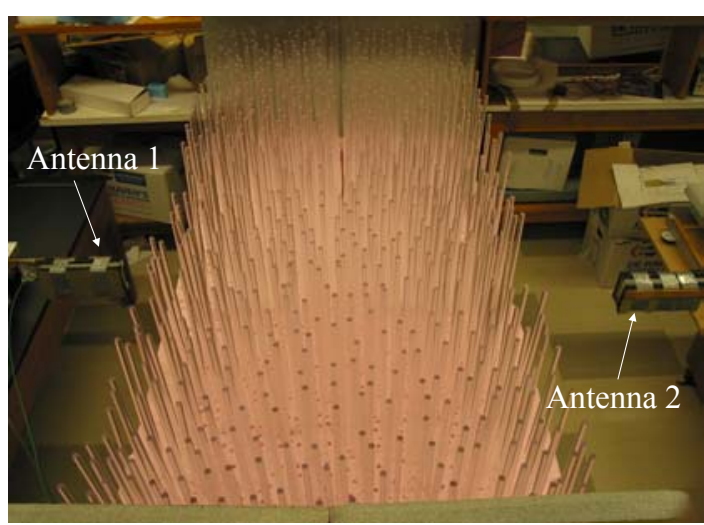

Figure 2. Dielectric rod experimental setup used in experimental time-reversal studies. The two antennas are moved using precision stepper motors. The length of the domain in the horizontal direction is $1.2 \mathrm{~m}$, and it is $2.4 \mathrm{~m}$ in the vertical direction (of this photo).

assumed that this ensemble captures the general properties for which the actual 
(unknown) forward-phase Green's function is a special case. Simple ideas from the signal-processing community are employed to improve time-reversal imaging quality under these assumptions.

\section{Experimental Configuration and Matched Media}

\section{A. Details of measurements}

Electromagnetic time reversal (ETR) measurements are performed using a vector network analyzer. The measurements are performed with Vivaldi antennas, over the 0.5$10.5 \mathrm{GHz}$ band. The electric fields are polarized vertically. Low-loss dielectric rods of $1.25 \mathrm{~cm}$ diameter, $0.6 \mathrm{~m}$ length, and with approximate dielectric constant $\varepsilon_{r}=2.5$ are situated with axes parallel to the direction of the electric fields. As indicated in Fig. 2, a total of 750 rods are considered, configured randomly, with an average inter-rod spacing of $6.5 \mathrm{~cm}$ (between rod axes). The rods are situated in a domain $1.2 \mathrm{~m}$ long and $2.4 \mathrm{~m}$ wide, with the rods embedded at the bottom in styrofoam $\left(\varepsilon_{r} \approx 1\right)$; the $2.4 \mathrm{~m}$ width is employed to minimize edge effects in the measurements, as detailed below. A top view of the geometry is shown in Fig. 3. The $1.2 \times 2.4 \mathrm{~m}^{2}$ rod domain is composed of six distinct and contiguous rod sections (see Fig. 3), each $2.4 \mathrm{~m}$ wide and $20 \mathrm{~cm}$ long. Multiple rod-placement realizations may be manifested by interchanging the positions of the six styrofoam-supported subsections. The antennas are situated in a plane bisecting the midpoint of the rods.

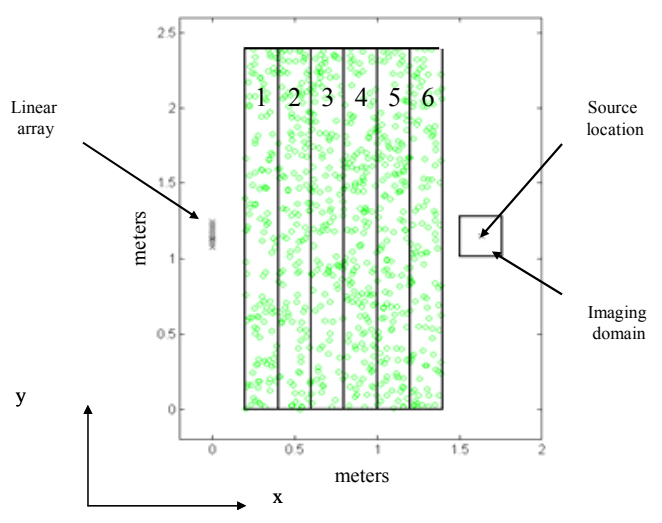

The measurements are performed with two antennas, one used for transmission and the other for reception. Multiple antenna

Figure 3. Top schematic view of the rods in Fig. 2. The rods are decomposed into six regions, with the rods in each region held together at the bottom via styrofoam. Different media instantiations are implemented by interchanging the positions of the six rod regions. 
positions are realized using precision stepper motors. The transmitting antenna, on one side of the domain (see Fig. 3), is placed at $M \times M$ positions, with inter-grid spacing $\Delta_{x}=\Delta_{y}=2.5 \mathrm{~cm}$. On the other side of the domain the receiver antenna is moved to $N$ positions along a linear aperture, with inter-element spacing $\Delta=2.5 \mathrm{~cm}$.

Let the transmitting-antenna location be represented as $\boldsymbol{r}_{m}$, for $m=1,2, \ldots, M^{2}$, and the receiving antenna is placed at $\boldsymbol{r}_{n}$, for $n=1,2, \ldots, N$. These $N M^{2}$ measurements constitute the data $G_{k}\left(\omega, \boldsymbol{r}_{n}, \boldsymbol{r}_{m}\right)$, where $\omega$ represents the angular frequency. As indicated above the positions of the six contiguous styrofoam sections are interchanged to constitute different media realizations, the $k$ th of which defining $G_{k}\left(\omega, \boldsymbol{r}_{n}, \boldsymbol{r}_{m}\right)$.

The center of the $M \times M$ domain is defined as the source position $\boldsymbol{r}_{s}$, and the fields measured on the linear aperture due to this source are $S_{k}\left(\omega, \boldsymbol{r}_{n}\right)=G_{k}\left(\omega, \boldsymbol{r}_{n}, \boldsymbol{r}_{m}=\boldsymbol{r}_{s}\right)$. An electromagnetic time-reversal (ETR) space-time image is computed using the measured Green's functions as

$$
I_{j k}\left(t, \boldsymbol{r}_{m}\right)=\sum_{n=1}^{N} \int_{\omega_{B W}} d \omega W^{*}(\omega) S_{j}^{*}\left(\omega, \boldsymbol{r}_{n}\right) G_{k}\left(\omega, \boldsymbol{r}_{n}, \boldsymbol{r}_{m}\right) \exp (-i \omega t)
$$

where the Fourier integral is performed over the system bandwidth $\omega_{B W}$, the symbol* denotes complex conjugate, and $W(\omega)$ is the window function used to weight (shape) the source excitation. While $S_{j}\left(\omega, \boldsymbol{r}_{n}\right)$ and $G_{k}\left(\omega, \boldsymbol{r}_{n}, \boldsymbol{r}_{m}\right)$ are both measured, the final image defined by (1) is synthesized; we typically consider the image manifested at $t=0$. We may observe ETR quality when the forward and inverse measurements are matched $(j=k)$ and when there is a mismatch $(j \neq k)$.

We note that the signals $S_{j}\left(\omega, \boldsymbol{r}_{n}\right)$ and $G_{k}\left(\omega, \boldsymbol{r}_{n}, \boldsymbol{r}_{m}\right)$ contain not only the Green's function of the medium, but also the responses of the antennas. The same two antennas are used for all transmission and reception measurements, and therefore the antenna response does not change the basic time-reversal principal. In addition, the transmitting 
and receiving antennas are on opposite ends of a highly cluttered media (the 750 dielectric rods) and therefore there is little if any direct coupling of the antennas.

\section{B. Time-reversal imaging for matched media: effective aperture}

In the first set of results we consider $j=k$ (matched media), and address image quality as a function of the bandwidth and aperture size. All images are shown at the $M \times M$ imaging points, at $t=0$, i.e., we plot $I_{j k}\left(t=0, \boldsymbol{r}_{m}\right)$. Ideally we expect spatial focusing at the center source location. In these examples $M=13$, and in the initial case $N=5$. A representative example image is presented in Fig. 4, for which tight spatial focusing is observed at the source location.

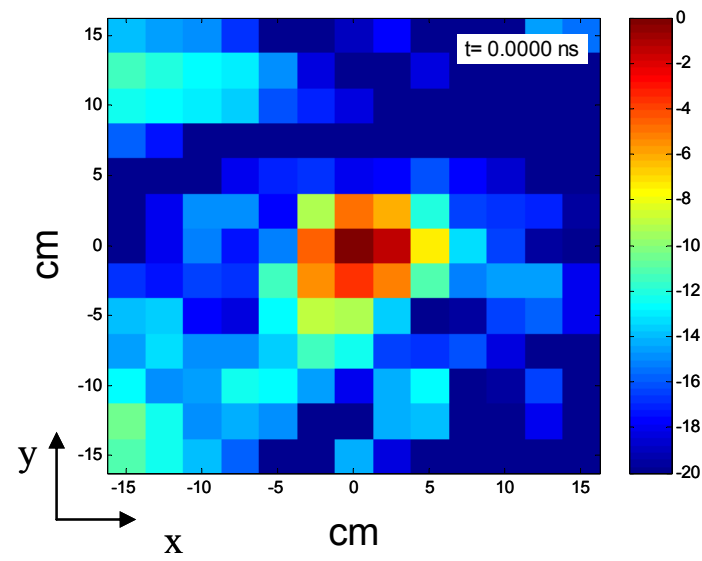

Figure 4. Time reversal focusing of a single antenna source via an $N=5$ element linear array. The color scale is in $\mathrm{dB}$. The results use the full $0.5-10.5 \mathrm{GHz}$ bandwidth.

The multi-path manifested from the rods yields "superresolution". It is of interest to examine this phenomenon in the context of the present measurements. Using the full-

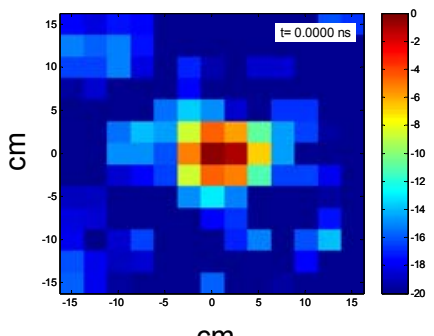

(a)

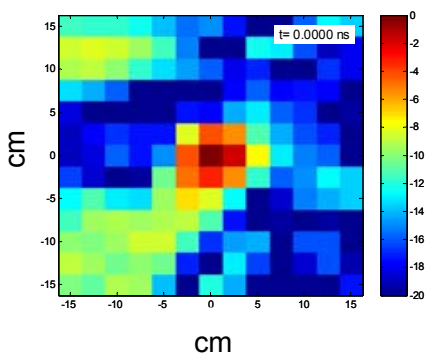

(b)

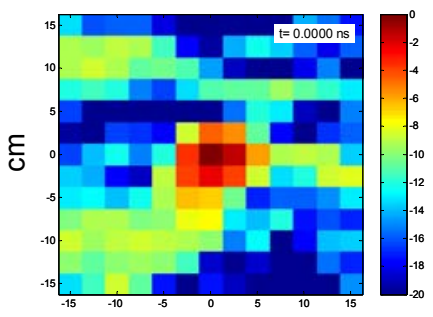

$\mathrm{cm}$

(c)

Figure 5. As in Fig. 4, but for $N=9, N=3$ and $N=1$ linear elements. (a) $N=9$, (b) $N=3$, (c) $N=1$

band data $(0.5-10.5 \mathrm{GHz})$ the measured cross-range resolution is approximately $7.5 \mathrm{~cm}$, with approximately $10 \mathrm{~cm}$ resolution in down-range. For a homogeneous medium, the 
anticipated cross-range resolution is $R_{c}=\lambda L / a$, where $\lambda$ is the wavelength, $a$ is the real linear aperture length, and $L$ is the distance from the aperture center to the imaging point. If we consider a free-space medium, and center frequency $5.5 \mathrm{GHz}$, a real aperture $a=10$ cm (for $N=5$ ), and length $L=1.5 \mathrm{~m}$, the optimal free-space cross-range resolution is $R_{c}=81.75 \mathrm{~cm}$; Fig. 4 demonstrates the significant improvement in cross-range resolution manifested by the rod-induced multipath.

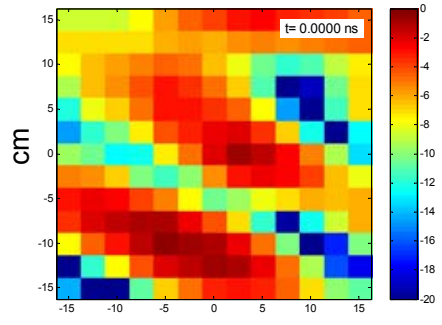

$\mathrm{cm}$

(a)

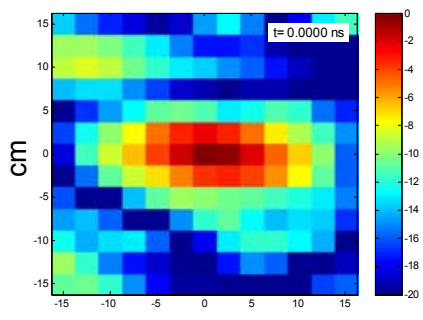

$\mathrm{cm}$

(b)

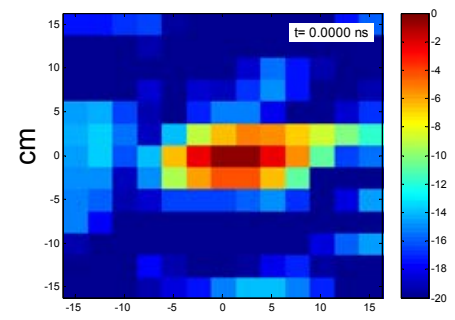

$\mathrm{cm}$

(c)

Figure 6. Time reversal focusing of a single antenna source via an $N=5$ element linear array. The color scale is in $\mathrm{dB}$. Results are shown using a constant bandwidth of $2.5 \mathrm{GHz}$, but with absolute bandwidths of 0.5-3 GHz, 2.5-5 GHz and 5-7.5 GHz. (a) 0.5-3 GHz, (b) $2.5-5 \mathrm{GHz}$, (c) 5-7.5 GHz.

To further assess the superresolution phenomenon, in Fig. 5 we present example timereversal imaging results (at $t=0$ ) for $N=9, N=3$ and $N=1$ receiver elements. The results for $N=5$ and $N=9$ elements are comparable, with only a slight degradation for the case of $N=3$.

\section{Time-reversal imaging for matched media: effect of bandwidth}

An important observation from our measurements is the dependence of the ETR image quality on the system bandwidth and the absolute frequencies employed, as was the case for acoustic waves [1,7]. For example, in Fig. 6 we consider $N=5$ linear elements and a fixed bandwidth of $2.5 \mathrm{GHz}$. However, the absolute frequencies considered in Figs. 6(a)(c) are respectively $0.5-3 \mathrm{GHz}, 2.5-5 \mathrm{GHz}$ and $5-7.5 \mathrm{GHz}$. The $5-7.5 \mathrm{GHz}$ results in Fig. 6(c) are only slightly less well focused than the full-band results in Fig. 4. However, much weaker focusing is observed for the bandwidth considered in Fig. 6(a). This is attributed to the fact that, at $3 \mathrm{GHz}$, the wavelength is relatively large relative to the inter- 
rod spacing and to the overall distance of propagation, and therefore the rods act as an effective mixture medium, rather than a highly multi-scattering environment. This lack of multipath undermines the aforementioned effective increase in the aperture size. However, the multipath increases for the 5-7.5

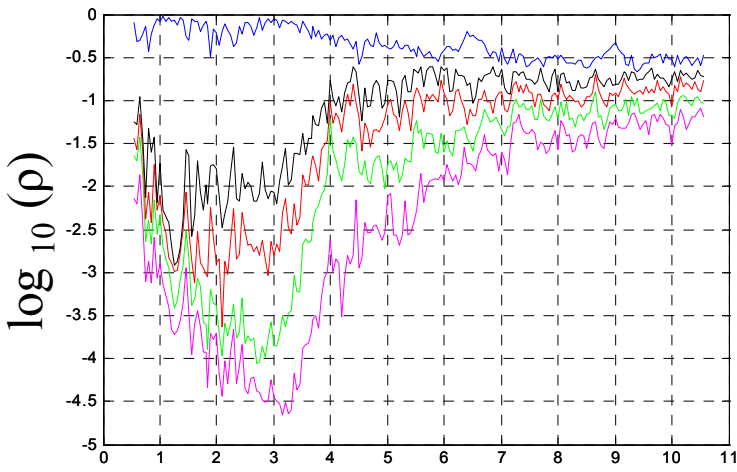

Frequency $(\mathrm{GHz})$

Figure 7. Logarithm of the five eigenvalues $\rho$ of the timereversal operator, as a function of frequency, for the data considered in Fig. 6.

$\mathrm{GHz}$ data, yielding multi-path-

induced superresolution.

To demonstrate the frequency-dependent properties of the dielectric-rod medium, let $\boldsymbol{v}_{j}(\omega)$ represent an $N \times 1$ vector, with $n$th element defined by $S_{j}\left(\omega, \boldsymbol{r}_{n}\right)$. The $N \times N$ time-

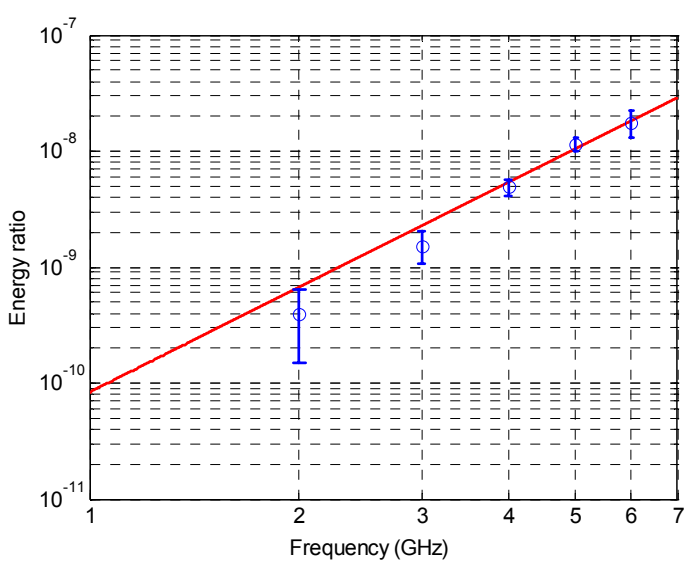

Figure 8. Mean focusing energy at source point, normalized by energy in excitation, as a function of frequency. The error bars represent the standard deviation across 24 realizations of the media, realized by interchanging the rod regions in Fig. 3. reversal array matrix [21] is defined as $\boldsymbol{V}(\omega)=\sum_{j=1}^{J} \boldsymbol{v}_{j}(\omega) \boldsymbol{v}_{j}(\omega)^{H}$, where we have summed over 24 realizations of the media (24 different orderings of the rods subsections in Fig. 2) and superscript $H$ denotes complex transpose. In Fig. 7 we plot the $N=5$ eigenvalues of $\boldsymbol{V}$ as a function of frequency (for $N=5$ linear receiver elements). If the medium is relatively weakly scattering, then each $\boldsymbol{v}_{j}(\omega)$ will be similar with small (random) variation, and one would expect a single principal eigenvalue, representative of the single source at $\boldsymbol{r}_{s}$. If the medium is highly scattering, waves will be incident upon the $N$-element receiver array from multiple 
angles, and the rank of $\boldsymbol{V}$ will increase. One notices two distinct regions in Fig. 7. Up to approximately $4 \mathrm{GHz}$ there is one principal mode, implying that in this frequency range the waves realize relatively weak multipath, and the rods constitute a relatively weakscattering random media. Above $4 \mathrm{GHz}$ the five eigenvalues are similar in magnitude, implying that $\boldsymbol{V}$ is full rank, and that the medium is highly scattering. As indicated in the previous paragraph, the highly-scattering character of the waves above $4 \mathrm{GHz}$ is beneficial to time reversal, in that it yields an increased effective aperture and superresolved imaging resolution.

\section{Frequency dependence of focusing energy, and image stability}

Theoretical studies in the Rayleigh scattering regime (scatterers of small diameter compared to wavelength) predicts that the ETR focusing intensity should increase as the third power of frequency in two space dimensions and as the fourth power of frequency in three dimensions [6] (as discussed in Sec. IIB). To consider this issue, we considered the ratio of energy focused at the original source location, normalized by the source energy, as a function of frequency. The experimental results in Fig. 8 confirm the theoretical predictions (for this approximately two-dimensional system), demonstrating a frequency dependence of $f^{3}$. The error bars in Fig. 8 correspond to different realizations of the media (as discussed in Sec. IIIA), manifested by interchanging the six rod regions, as reflected in Fig. 3). To compute the results in Fig. 8, full-band ETR imaging was performed, yielding a time-dependent signal for the data imaged via time reversal to the original source location. A Fourier transform was performed of this waveform, and the energy was normalized to the strength of the excitation energy, as a function of frequency.

We also note that the error bars reflected in Fig. 8, corresponding to variation in $I_{j j}\left(t=0, \boldsymbol{r}_{m}\right)$ for 24 distinct manifestations of the intervening rod media, are relatively tight (little variation across different media realizations). This observation is consistent with previous studies that predict a high degree of stability $[4,20,21]$ in the time-reversal image quality, for different media realizations (assuming the media used in the forward 
and inverse steps is the same). Note as well that the error bars get tighter with increasing frequency, this corresponding to more cluttered media (relative to the decreasing wavelength) and hence more multi-path.

\section{Controlled Mismatched Media and Comparisons to Theory}

The following measurements are motivated by theoretical studies of time-reversal in changing media. In Fig. 9 we consider the $2.4 \mathrm{~m}$ wide and $1.2 \mathrm{~m}$ long media discussed above. A source on the right of the domain emits energy that is observed at the $N=5$ linear elements at left (the source is at the midpoint of the $2.4 \mathrm{~m}$ rod-region width). The data are time reversed (phase conjugated) and the measured Green's function over the square imaging domain is used to constitute an ETR image at $t=0$, as in (1). We now consider how the ETR image changes if the time-reversed fields are imaged using a Green's function corresponding to the shifted media in Fig. 9 (now $j \neq k$ in (1); this corresponds to the case discussed in Sec. IID). By incrementally increasing the shift through which ETR imaging is performed (Fig. 9), we perform a controlled examination of how ETR

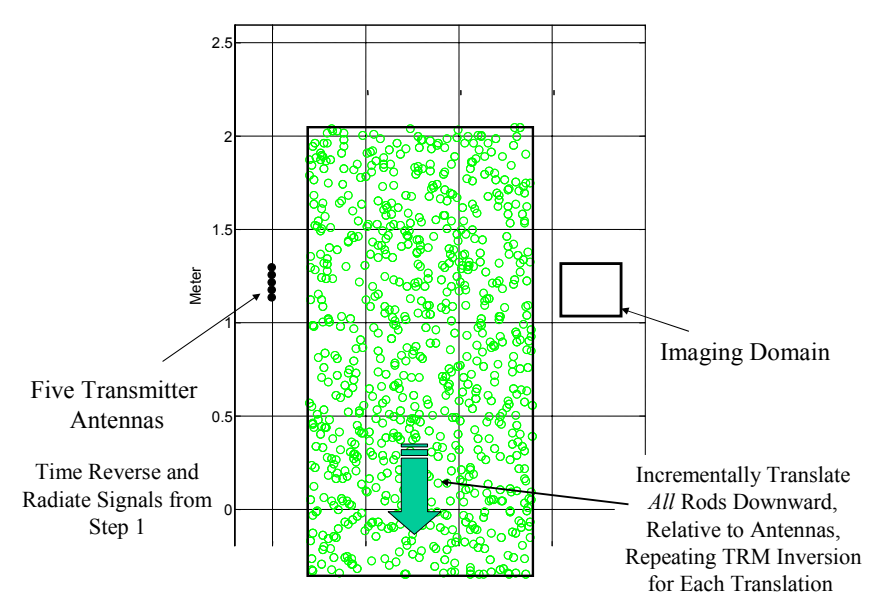

Figure 9. Examination of time reversal imaging in changing media, with change manifested via a shift in the media. In Step 1 a single antenna is placed in the center of the imaging domain at right, and the radiated fields are observed on the five antennas at left. These fields are time reversed and reradiated into the domain, with the fields from this process observed in the imaging domain. Imaging results are considered as a function of the relative shift in the media, considered within the time-reversal stage. refocusing quality deteriorates as the mismatch (shift distance) increases between the original and shifted (Fig. 9) media used for imaging. Note that the use of a $2.4 \mathrm{~m}$ wide media was necessitated by edge effects observed when executing such shifting when the media was only $1.2 \mathrm{~m}$ wide.

The ETR images at $t=0$ are shown in Fig. 10, as a function of shift distance. The white circle in each sub-image of Fig. 10 
corresponds to the physical location of the actual original source, with respect to which the imaging domain is shifted (see Fig. 9). Considering Fig. 10, we note that the downrange resolution (along the horizontal direction of the figures) is preserved as the media is shifted; however, with increasing shift distance the cross-range localization (vertical direction) is lost.

As reviewed in Sec. IID, in the diffusion regime, it has been predicted that the amplitude at the center of the imagery domain, for shifts of the type considered in Fig. 10, should vary as $J_{o}(k \tau)$ where $\tau$ is the spatial shift and $k$ is the wavenumber. A qualitatively similar result is predicted in the radiative transfer regime. In Fig. 11 we plot the energy observed at the center of each image, as a function of the shift between the media considered in step (i) and step (ii) of the time-reversal process. In addition, the results in Fig. 11 are presented as a function of the bandwidth used when performing time reversal. In Fig. 12 we plot the magnitude of the excitation window used in $(1),|W(\omega)|$. Considering $|W(\omega)|$, the strongest energy in one of the subbands considered in Fig. 12 occurs at the respective peak energy (e.g., for the $0.5-1 \mathrm{GHz}$ subband, the greatest energy
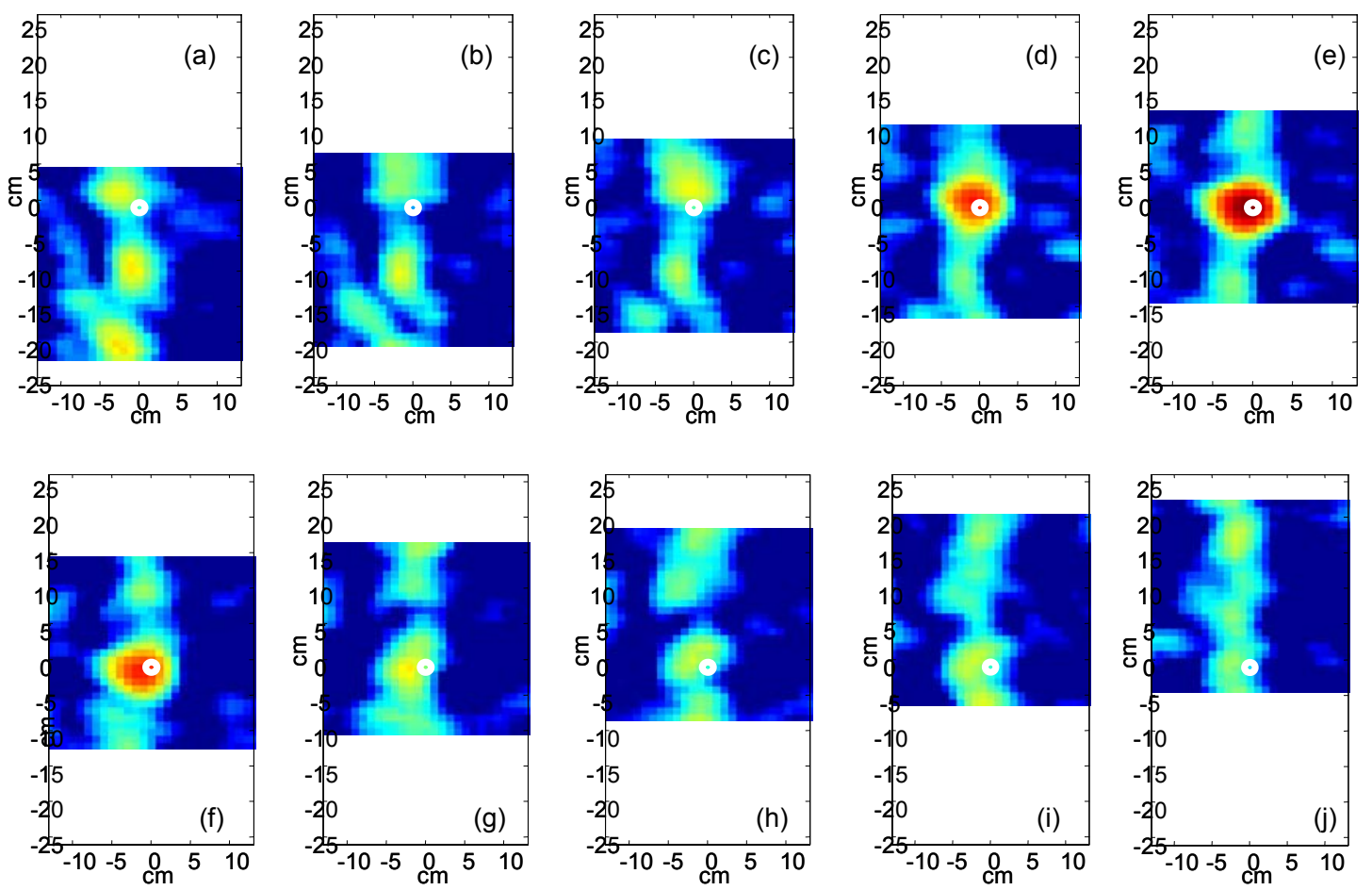

Figure 10. Time reversal image as a function of shift in the media (see Fig. 9). The position of the original source is shown with the white circle. (a) $-8 \mathrm{~cm}$ shift, (b) $-6 \mathrm{~cm}$, (c) $-4 \mathrm{~cm}$, (d) $-2 \mathrm{~cm}$, (e) $0 \mathrm{~cm}$, (f) $2 \mathrm{~cm}$, (g) $4 \mathrm{~cm}$, (h) $6 \mathrm{~cm}$, (i) $8 \mathrm{~cm}$, (j) $10 \mathrm{~cm}$ 
is at $1 \mathrm{GHz})$.

Using $J_{o}(k \tau)$, the first null in the predicted energy at the center of the imaging domain should occur at shifts of $12 \mathrm{~cm}, 8 \mathrm{~cm}, 6 \mathrm{~cm}$ and 4.8 $\mathrm{cm}$, for respective (peak) frequencies of $1 \mathrm{GHz}, 1.5 \mathrm{GHz}, 2 \mathrm{GHz}$ and 2.5 GHz. By considering Fig. 12, we note excellent agreement between

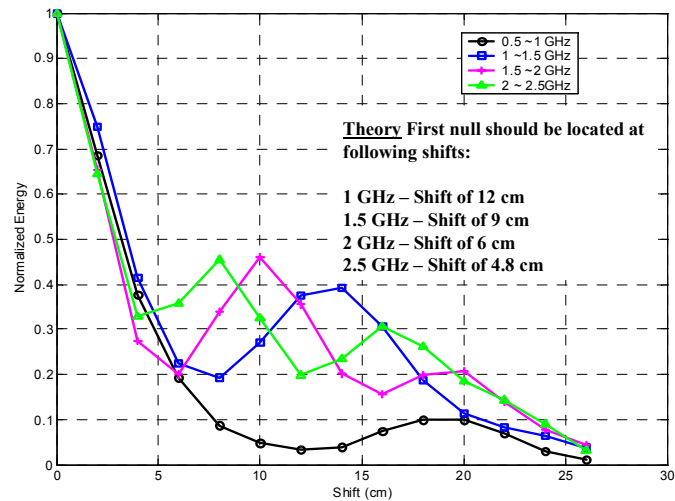

Figure 11. Normalized energy at the center of each subimage in Fig. 10, as a function of sensor bandwidth. The theoretical predictions for the position of the first null are tabulated in the inset.

our measured results and the predictions based on the theory in [6]. A qualitative behavior of the refocused intensity proportional to $J_{o}(k \tau)$ is not only a characteristic of

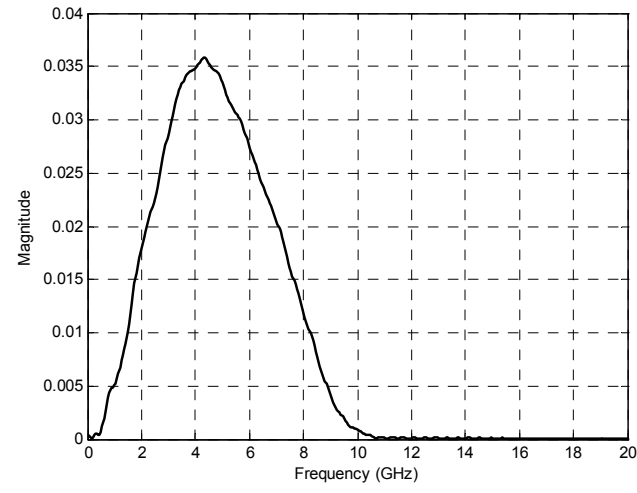

Figure 12. Spectrum of the waveform used in the time-reversal experiments.

the diffusion regime; for instance it approximately holds in the less scattering radiative transfer regime [6]. We have also stressed that frequencies below $4 \mathrm{GHz}$ were weakly scattering. However it does demonstrate that multiple scattering is responsible for the refocused signal, for in homogeneous medium, the refocused energy would not depend on the $\operatorname{shift} \tau$. Moreover it indicates that loss of correlation between the two media (see Sec. IIC) of the two stages of the time reversal experiment induces reduction in refocused signal strength.

\section{Imaging in Changing Media}

\section{A. Imaging with mismatched Green's function}

We consider image quality $I_{j k}\left(t=0, \boldsymbol{r}_{m}\right)=\sum_{n=1}^{N} \int_{\omega_{B W}} d \omega S_{j}^{*}\left(\omega, \boldsymbol{r}_{n}\right) G_{k}\left(\omega, \boldsymbol{r}_{n}, \boldsymbol{r}_{m}\right)$ for $j \neq k$, addressing the problem for which there is a completely distinct arrangement of the six 
regions for cases $j$ and $k$ (see Fig. 3). Specifically, for the $j \neq k$ cases considered here there is no overlap for any of the six rod subsections with respect to media realizations $j$ and $k$ (complete mismatch). The image is computed using the discrete sampled frequencies as

$$
I_{j k}\left(t=0, \boldsymbol{r}_{m}\right)=\sum_{n=1}^{N} \boldsymbol{s}_{j}^{H}(n) \boldsymbol{g}_{k}(n, m)
$$

where $\boldsymbol{s}_{j}(n)$ is an $N_{F} \times 1$ column vector representing the frequency dependence of $S_{j}\left(\omega, \boldsymbol{r}_{n}\right), \boldsymbol{g}_{k}(n, m)$ is an $N_{F} \times 1$ column vector representing the frequency dependence of $G_{k}\left(\omega, \boldsymbol{r}_{n}, \boldsymbol{r}_{m}\right), N_{F}$ is the number of frequencies considered, and superscript $H$ represents conjugate transpose.

\section{B. Averaged Green's function}

Assume we have measured data $S_{j}\left(\omega, \boldsymbol{r}_{n}\right)$ from the linear array of receivers, corresponding to the $j$ th realization of the media (placement of the six rod subsections). Further, assume that the particular media (Green's function) associated with this $j$ th example is unknown. However, we may have access to the statistics of the Green's function. Specifically, assume access to Green's functions from $K$ realizations of the media ( $K$ realizations of the six rod subsections in Fig. 3), each of which is entirely distinct from the $j$ th realization referenced above (as discussed above). If these $K$ Green's functions are denoted $G_{k}\left(\omega, \boldsymbol{r}_{n}, \boldsymbol{r}_{m}\right)$, for $k=1, \ldots, K$, then one simple idea would be to perform imaging using the average Green's function

$$
G_{A V G}\left(\omega, \boldsymbol{r}_{n}, \boldsymbol{r}_{m}\right)=\frac{1}{K} \sum_{k=1}^{K} G_{k}\left(\omega, \boldsymbol{r}_{n}, \boldsymbol{r}_{m}\right)
$$

with the time-reversal image represented as

$$
I_{j, A V G}\left(t=0, \boldsymbol{r}_{m}\right)=\sum_{n=1}^{N} \boldsymbol{s}_{j}^{H}(n) \boldsymbol{g}_{A V G}(n, m)
$$

where $\boldsymbol{g}_{A V G}(n, m)$ is an $N_{F} \times 1$ column vector representing the frequency dependence of $G_{A V G}\left(\omega, \boldsymbol{r}_{n}, \boldsymbol{r}_{m}\right)$. 


\section{Subspace imaging}

For a given source and receiver point, again assume access to $K$ examples of the Green's function, denoted $G_{k}\left(\omega, \boldsymbol{r}_{n}, \boldsymbol{r}_{m}\right)$, for $k=1, \ldots, K$. Rather than taking the average of these $K$ Green's functions (for $K$ realizations of the media), we may perform a principal components analysis (PCA) [18], and project the measured data onto the principal Green's function eigenvectors. We perform an eigen decomposition of the $N_{F} \times N_{F}$ matrix

$$
\boldsymbol{G}(n, m)=\sum_{k=1}^{K} \boldsymbol{g}_{k}(n, m) \boldsymbol{g}_{k}(n, m)^{H}
$$

Let $\boldsymbol{e}_{l}(n, m)$ represent the $l$ th eigenvector of (5). A PCA-based imaging result is represented as

$$
I_{j, E I G}\left(t=0, \boldsymbol{r}_{m}\right)=\sum_{n=1}^{N} \sum_{l=1}^{L_{P}} \boldsymbol{s}_{j}^{H}(n) \boldsymbol{e}_{l}(n, m)
$$

In the example results we have considered $L_{p}=1$ principal component.

\section{Example imaging results}

The measurements were performed as follows. The Green's function $G\left(\omega, \boldsymbol{r}_{n}, \boldsymbol{r}_{m}\right)$ was measured for $N_{\text {media }}=24$ realizations of the six rod subsections depicted in Fig. 3. For the $j$ th of these realizations, there was a set $\Omega_{j}$ of $N_{j}<N_{\text {media }}$ other rod arrangements considered for which there was a complete mismatch in the order of the six rod regions.

When considering the technique in Sec. IVA for media $j, I_{j k}$ was calculated separately for all media $k \in \Omega_{j}$; consequently, for each media $j$ we compute $N_{j}$ distinct images $I_{j k}$. For the techniques in Secs. IVB and IVC, a single image is computed for each of the $N_{\text {media }}$ media arrangements. Specifically, for $I_{j, A V G}$ the average Green's function $\boldsymbol{g}_{A V G}(n, m)$ was computed using the $N_{j}$ media in $\Omega_{j}$; for $I_{j, E I G}$ the principal component $\boldsymbol{e}_{1}(n, m)$ was also computed using the $N_{j}$ media in $\Omega_{j}$. In these examples a total of $N_{\text {media }}=24$ media were considered, and for each media $j$ on average $N_{j}=10$ (the minimum was $N_{j}=6$ ). 
In Fig. 13(a) a representative image is performed using traditional time reversal as in (2), for which the Green's functions used for the forward and inverse parts of time reversal are entirely distinct - corresponding to (2). Figures 13(b) as 13(c) present representative results for $I_{j, E I G}\left(t=0, \boldsymbol{r}_{m}\right)$ and $I_{j, A V G}\left(t=0, \boldsymbol{r}_{m}\right)$, respectively, for which generally good imaging is observed. The improved results of Figs. 13(b) and 13(c) vis-à-vis Fig. 13(a) is expected, given that the former employ more information. However, we underscore that

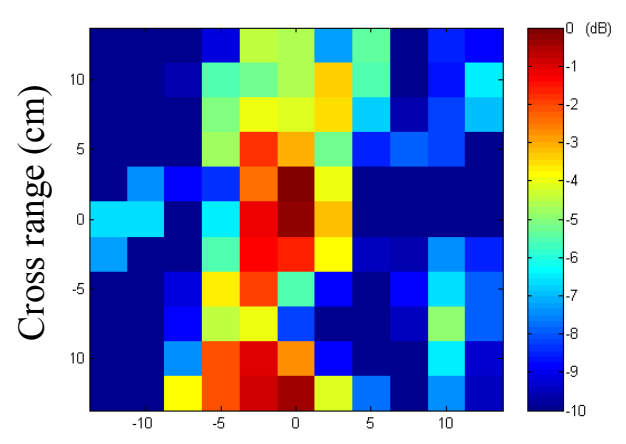

Down range $(\mathrm{cm})$

(a)

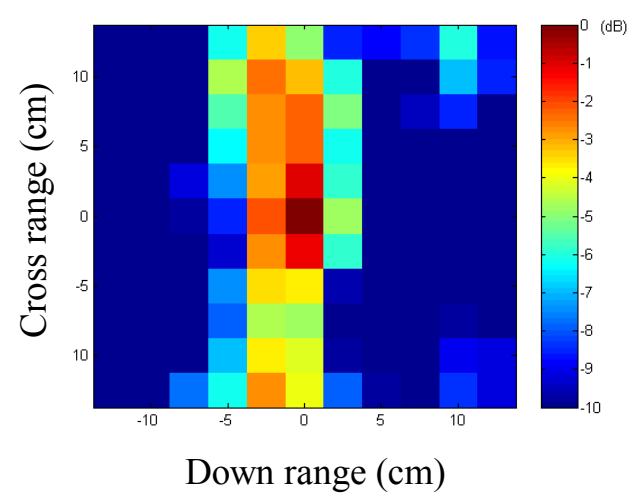

(c)

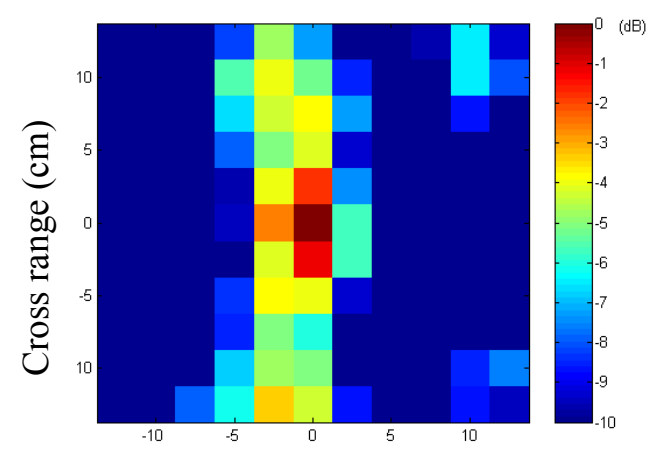

Down range $(\mathrm{cm})$

(b)

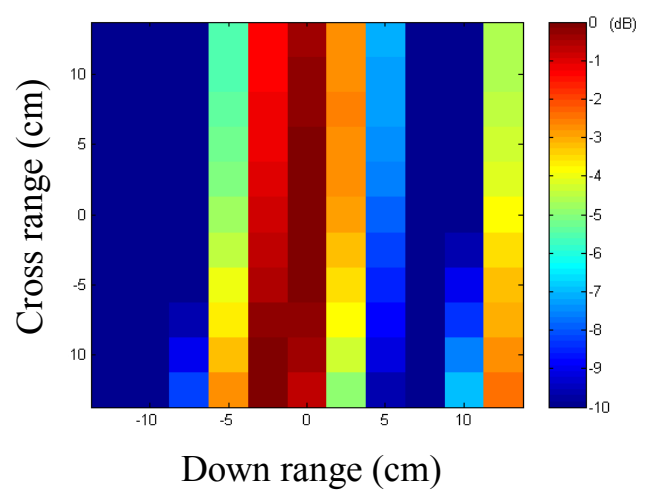

(d)

Figure 13. Typical time-reversal images for the case in which there is a complete mismatch between the Green's function associated with the forward and inverse phases of time reversal. Results are on a $10 \mathrm{~dB}$ scale. (a) Using mismatched Green's function, (b) using an eigen Green's function, (c) using an average Green's function, (d) using the free-space Green's function.

each of Green's function employed in Figs. 13(a), 13(b) and 13(c), for computation of the average and eigen Green's functions, are entirely distinct from media $j$ associated with forward measured data $S_{j}\left(\omega, \boldsymbol{r}_{n}\right)$. 


\section{F. Quantitative analysis of imaging results}

It is of interest to perform a quantitative analysis of all analyzed images of the form in Fig. 13. Toward this end, a square region is defined corresponding to a contiguous $3 \times 3$ pixel region in image space, with pixels defined as in Fig. 13. The test statistic $l$ used in this analysis is the peak pixel amplitude within the $3 \times 3$ pixel box. We compute this test statistic $l$ for all possible $3 \times 3$ boxes within the image. For a general threshold $T$, if $l>T$ a target is declared as being within the corresponding $3 \times 3$ inner

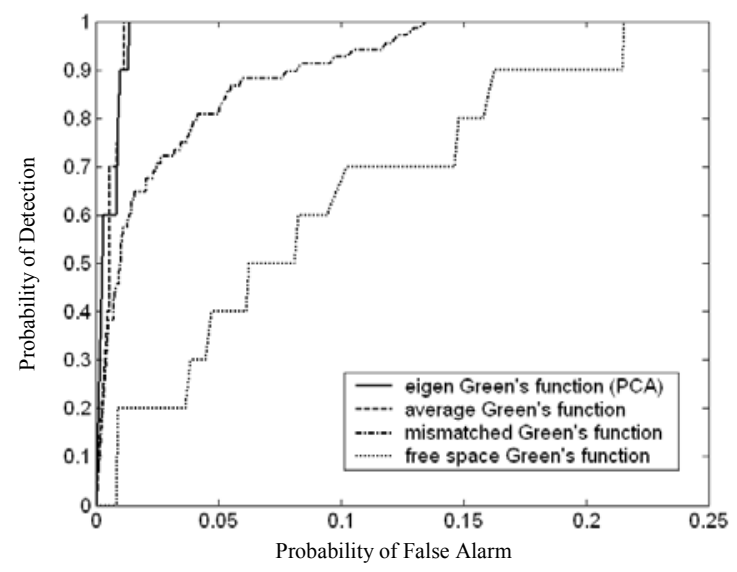

Figure 14. Receiver operating characteristic (ROC) averaged across multiple realizations of mismatched media, using the four imaging techniques reflected in Fig. 13. region, and if $l<T$ no target is declared (the actual target position is in the center of the image, as in Fig. 13). By varying the threshold $T$, from zero to $T>l_{\max }$ (the largest value of $l$ within a given image), we yield the receiver operating characteristic (ROC) [22], representing the probability of detection as a function of the probability of false alarm (a false alarm is defined by declaring the presence of a source at a location for which there is in reality none). In Fig. 14 we plot the ROC curves for the imaging techniques considered in Fig. 13. We note that the techniques based on the average and eigen Green's functions (equations (4) and (6), respectively) yield comparable performance, while performance deteriorates precipitously when considering a single mismatched Green's function or the free-space Green's function (the latter yielding the worst results).

\section{Conclusions}

Time-reversal imaging has been examined experimentally for electromagnetic source localization in highly scattering media. We initially addressed the well-known time- 
reversal behavior for the case in which the media employed in the forward and inverse steps are matched. These experiments confirmed theoretical predictions concerning timereversal imaging stability, for the case in which the forward and inverse media are matched, but for different media realizations [6]. The experiments also confirmed the anticipated $f^{3}$ frequency dependence of the imaging amplitude in the Rayleigh regime [6], for the case of matched media.

In our first analysis of time-reversal imaging in changing media, we considered the special case for which the two media are shifted with respect to each other. While this is a special case, it is of particular interest because it allows us to address the accuracy of previous theoretical predictions [6]. In particular, in the diffusive regime it is predicted that the imaged amplitude should vary as $J_{o}(k \tau)$, where $k$ represents the wavenumber and $\tau$ the spatial shift [6]; the experimental results considered here are in excellent agreement with theoretical predictions.

While the theory and experimental results indicate time-reversal imaging deterioration as media mismatch increases between the forward and inverse phases, we have also considered alternative techniques for the case in which the media employed in the forward phase is either unknown or may not be modeled precisely. Specifically, we have performed time-reversal imaging based on an average Green's function, computed using an ensemble of Green's functions; each Green's function in the ensemble corresponds to a media completely distinct from that used in the forward step, but with similar statistics. A similar use of the ensemble of Green's functions was considered via an eigen analysis, analogous to principal components analysis (PCA) [18]. It was demonstrated that we often observe significantly improved time-reversal imaging quality based on either the average or eigen Green's function (quantified in terms of the receiver operating characteristic, or ROC).

While the ensemble of Green's functions were employed to improve imaging performance, there remains a question of how this could be utilized in practice. If one is performing the inverse phase using computed Green's functions, there may be 
uncertainty as to which specific propagation media should be used within the computational model. The results presented here suggest that one may consider an ensemble of computed Green's functions within the inverse phase, with this ensemble designed to capture the range of different media deemed representative of the forward measurement. In the results presented here, the Green's functions were measured, for different realizations of the intervening media. If one wished to perform time-reversal imaging in a multi-path environment, such as an urban setting, the Green's functions could be measured, analogous to wireless measurements. The variable properties of such a propagation environment may be captured by an ensemble of Green's functions, measured at different times, as the propagation media changes.

\section{References}

[1] M. Fink, "Time-reversal of ultrasonic fields - Part I: Basic Principles," IEEE Trans. Ultrason., Ferroelectr., and Freq. Control, vol 39, pp. 555-566, 1992

[2] B. Steinberg, Microwave Imaging Techniques, New York: J. Wiley, 1991

[3] D.R. Jackson and D.R. Dowling, "Phase conjugation in underwater acoustics," $J$. Acoust. Soc. of Amer., vol. 89, pp. 171-181,1990

[4] P. Blomberg, G. Papanicolaou, and H.K. Zhao, "Super-resolution in time-reversal acoustics," J. Acoust. Soc. of Amer., vol. 111, pp. 230-248, 2002

[5] G. Bal and L. Ryzhik, "Time reversal and refocusing in random media," SIAM Appl. Math., vol. 63 (5), pp. 1375-1498, 2003

[6] G. Bal and R. Verastegui, "Time reversal in changing environment," Multiscale Model. Simul. , vol. 2, pp. 639-661, 2004

[7] M. Fink, "Time reversed acoustics," Physics Today, 50, pp. 34-40, 1997

[8] A. Derode, P. Roux, and M. Fink, "Robust acoustic time reversal with high-order multiple scattering," Phys. Rev. Lett., vol. 75, pp. 4206-4209, 1995

[9] P. Roux, B. Roman and M. Fink, "Time-reversal in an ultrasonic waveguide," Appl. Phys. Letts., vol. 70, 1997 
[10] J. de Rosny, A. Tourin, A. Derode, B. van Tiggelen and M. Fink, "The relation between time reversal focusing and coherent backscattering in multiple scattering media: a diagrammatic approach," Phys. Rev. E, vol. 70, 046601, 2004

[11] W.A. Kuperman, W.S. Hodgkiss, H.C. Song, T. Akal, C. Ferla, and D.R. Jackson, "Phase conjugation in the ocean: Experimental demonstration of an acoustic timereversal mirror," J. Acoust. Soc. Am., vol. 103, pp. 25-40, 1998.

[12] H.C. Song, W.A. Kuperman, W.S. Hodgkiss, T. Akial and C. Ferla, "Iterative time reversal in the ocean," J. Acoust. Soc. Am., vol. 105, pp. 3176-3184, 1999.

[13] S. Kim, G.F. Edelmann, W.A. Kuperman, W.S. Hodgkiss, H.C. Song and T. Akal, "Spatial resolution of time-reversal arrays in shallow water," J. Acoust. Soc. Am., vol. 110, pp. 820-829, 2001.

[14] G. Lerosey, J. de Rosny, A. Tourin, A. Derode, G. Montaldo and M. Fink, "Time reversal of electromagnetic waves," Phys. Rev. Letts., vol. 92(19), 2004

[15] D. Liu, G. Kang, L. Li, Y. Chen, S. Vasudevan, W. Joines, Q. H. Liu, J. Krolik and L. Carin, "Electromagnetic time-reversal imaging of a target in a cluttered environment," IEEE Trans. Antenna Propag., vol. 53, pp. 3058-3066, 2005

[16] J.L. Krolik, "The performance of matched-field beamformers with Mediterranean vertical array data," IEEE Trans. Signal Process., vol. 44, pp. 2605-2611, 1996

[17] J.L. Krolik, "Matched-field minimum variance beamformaing in a random ocean channel," J. Acoust. Soc. Amer., vol. 92, pp.1408-1419, 1992

[18] A. Basilevsky, Statistical Factor Analysis and Related Methods, Theory and Application. New York: J. Wiley, 1994

[19] A. Ishimaru, Waves Propagation in Scattering and Random Media, Wiley-IEEE Press, 1999.

[20] G. Bal, "On the self-averaging of wave energy in random media," SIAM Multiscale Model. Simul. , vol. 2, pp. 398-420, 2004

[21] L. Borcea, G. Papanicolaou, C. Tsogka and J. Berryman, "Imaging and time reversal in random media," Inverse Problems, vol. 18, pp. 1247-1279, 2002

[22] T.D. Wickens, Elementary Signal Detection Theory. New York: Oxford University Press, 2002. 